\title{
Metrics on the Sets of Nonsupersingular Elliptic Curves in Simplified Weierstrass Form over Finite Fields of Characteristic Two
}

\author{
Keisuke Hakuta \\ Interdisciplinary Graduate School of Science and Engineering, Shimane University, 1060 Nishikawatsu-cho, \\ Matsue-shi, Shimane 690-8504, Japan \\ Correspondence should be addressed to Keisuke Hakuta; hakuta@cis.shimane-u.ac.jp
}

Received 25 August 2015; Accepted 19 November 2015

Academic Editor: Aloys Krieg

Copyright ( 2015 Keisuke Hakuta. This is an open access article distributed under the Creative Commons Attribution License, which permits unrestricted use, distribution, and reproduction in any medium, provided the original work is properly cited.

\begin{abstract}
Elliptic curves have a wide variety of applications in computational number theory such as elliptic curve cryptography, pairing based cryptography, primality tests, and integer factorization. Mishra and Gupta (2008) have found an interesting property of the sets of elliptic curves in simplified Weierstrass form (or short Weierstrass form) over prime fields. The property is that one can induce metrics on the sets of elliptic curves in simplified Weierstrass form over prime fields of characteristic greater than three. Later, Vetro (2011) has found some other metrics on the sets of elliptic curves in simplified Weierstrass form over prime fields of characteristic greater than three. However, to our knowledge, no analogous result is known in the characteristic two case. In this paper, we will prove that one can induce metrics on the sets of nonsupersingular elliptic curves in simplified Weierstrass form over finite fields of characteristic two.
\end{abstract}

\section{Introduction}

Elliptic curves have been studied by number theorists for a very long time. Nowadays, elliptic curves have been the focus of much attention due to not only the theoretical aspects but also the practical aspects in computational number theory. In particular, elliptic curves have a wide variety of applications in computational number theory such as elliptic curve cryptography $[1,2]$, pairing based cryptography $[3,4]$, primality tests $[5,6]$, and integer factorization $[7,8]$.

Mishra and Gupta in [9] have found an interesting property of the sets of elliptic curves in simplified Weierstrass form (or short Weierstrass form) over prime fields of characteristic greater than three. The property is that one can induce metrics on the sets of elliptic curves in simplified Weierstrass form over prime fields of characteristic greater than three. Later, Vetro in [10] has found some other metrics on the sets of elliptic curves in simplified Weierstrass form over prime fields of characteristic greater than three. They have proposed potential applications of the metrics to the protection of side channel attacks [11]. However, to our knowledge, no analogous result is known in the characteristic two case. In this direction, it seems mathematically natural to explore a methodology for constructing metrics on (sub)sets of elliptic curves over finite fields of characteristic two whether there is a cryptographic application or not.

The motivation of this work is to study the characteristic two case. We will prove that one can induce metrics on the sets of nonsupersingular elliptic curves in simplified Weierstrass form over finite fields of characteristic two.

The rest of this paper is organized as follows. In Section 2, we recall some basic facts that will be used throughout the paper. In Section 3, we give metrics on the sets of nonsupersingular elliptic curves in simplified Weierstrass form over finite fields of characteristic two. Section 4 concludes the paper.

\section{Mathematical Preliminaries}

In this section we fix our notation and recall some basic facts that will be used throughout the paper. For more details, we refer the reader to [12, Section 3.3], [13, Appendix A]. 
Let $K$ be a field. For any field $K$, we denote by $p=\operatorname{char}(K)$ the characteristic of the field $K$. We use the symbols $\mathbb{Z}, \mathbb{R}$, and $\mathbb{F}_{q}$ to represent the integers, real numbers, and a finite field with $q$ elements, where $q=p^{r}(r \geq 1), p=\operatorname{char}\left(\mathbb{F}_{q}\right)$. For a finite set $S$, we denote the cardinality of $S$ by $\sharp S$.

Let $E / K: y^{2}+a_{1} x y+a_{3} y=x^{3}+a_{2} x^{2}+a_{4} x+a_{6}$ be an algebraic curve over $K$. We set

$$
\begin{aligned}
d_{2} & =a_{1}^{2}+4 a_{2}, \\
d_{4} & =2 a_{4}+a_{1} a_{3}, \\
d_{6} & =a_{3}^{2}+4 a_{6}, \\
d_{8} & =a_{1}^{2} a_{6}+4 a_{2} a_{6}-a_{1} a_{3} a_{4}+a_{2} a_{3}^{2}-a_{4}^{2}, \\
c_{4} & =d_{2}^{2}-24 d_{4}, \\
\Delta & =-d_{2}^{2} d_{8}-8 d_{4}^{3}-27 d_{6}^{2}+9 d_{2} d_{4} d_{6}, \\
j(E) & =\frac{c_{4}^{3}}{\Delta} .
\end{aligned}
$$

The next theorem provides a necessary and sufficient condition that $E / K$ is an elliptic curve.

Theorem 1 (see [12, Theorem 2.4]). E is an elliptic curve; that $i$, the Weierstrass equation is nonsingular, if and only if $\Delta \neq 0$.

Two elliptic curves $E_{1}$ and $E_{2}$ are called isomorphic if there exist morphisms (as algebraic varieties) from $E_{1}$ to $E_{2}$ and from $E_{2}$ to $E_{1}$ which are inverses of each other. The following theorems (Theorems 2 and 3) tell us when two elliptic curves are isomorphic.

Theorem 2 (see [12, Theorem 2.5]). If two elliptic curves $E_{1} / K$ and $E_{2} / K$ are isomorphic over $K$, then $j\left(E_{1}\right)=j\left(E_{2}\right)$. The converse is also true if $K$ is an algebraically closed field.

Theorem 3 (see [12, Theorem 2.1]). Two elliptic curves $E_{1} / K$ and $E_{2} / K$ given by the equations

$$
\begin{aligned}
& E_{1}: y^{2}+a_{1} x y+a_{3} y=x^{3}+a_{2} x^{2}+a_{4} x+a_{6}, \\
& E_{2}: y^{2}+\bar{a}_{1} x y+\bar{a}_{3} y=x^{3}+\bar{a}_{2} x^{2}+\bar{a}_{4} x+\bar{a}_{6}
\end{aligned}
$$

are isomorphic over $K$ (or $K$-isomorphic), denoted by $E_{1} / K \cong$ $E_{2} / K$, if and only if there exists $u, r, s, t \in K, u \neq 0$, such that the change of variables

$$
(x, y) \longmapsto\left(u^{2} x+r, u^{3} y+u^{2} s x+t\right)
$$

transforms equation $E_{1}$ to equation $E_{2}$. The relationship of isomorphism is an equivalence relation.

Theorem 4 is the famous Hasse bound for the number of rational points on elliptic curves over finite fields.

Theorem 4 (Hasse). Let $\sharp E\left(\mathbb{F}_{q}\right)=q+1-t$. Then $|t| \leq 2 \sqrt{q}$.

The elliptic curve $E / \mathbb{F}_{q}$ is called supersingular if $p$ divides t. Otherwise, the curve $E / \mathbb{F}_{q}$ is called nonsupersingular (or ordinary). It is well-known that if $p=2$ or $p=3$, then $E$ is supersingular if and only if $j(E)=0$. In other words, if $p=2$ or $p=3, E$ is nonsupersingular if and only if $j(E) \neq 0$. Remark that if $E / K: y^{2}+\bar{a}_{1} x y+\bar{a}_{3} y=x^{3}+\bar{a}_{2} x^{2}+\bar{a}_{4} x+\bar{a}_{6}$ is an elliptic curve with $\operatorname{char}(K)=2$ and $j(E) \neq 0$, then the admissible change of variables

$$
(x, y) \longmapsto\left(\bar{a}_{1}^{2} x+\frac{\bar{a}_{3}}{\bar{a}_{1}}, \bar{a}_{1}^{3} y+\frac{\bar{a}_{1}^{2} \bar{a}_{4}+\bar{a}_{3}^{2}}{\bar{a}_{1}^{3}}\right)
$$

transforms $E$ to the nonsupersingular elliptic curve

$$
E^{\prime} / K: y^{2}+x y=x^{3}+a_{2} x^{2}+a_{6} \text {. }
$$

An elliptic curve of form (5) is called simplified Weierstrass form (or short Weierstrass form). Let $E_{1} / \mathbb{F}_{2^{m}}, E_{2} / \mathbb{F}_{2^{m}}$

$$
\begin{array}{ll}
E_{1}: y^{2}+x y=x^{3}+a_{2} x^{2}+a_{6} & \left(a_{6} \neq 0\right), \\
E_{2}: y^{2}+x y=x^{3}+\bar{a}_{2} x^{2}+\bar{a}_{6} & \left(\bar{a}_{6} \neq 0\right)
\end{array}
$$

be nonsupersingular elliptic curves over $\mathbb{F}_{2^{m}}$ in simplified Weierstrass form. If $E_{1} / \mathbb{F}_{2^{m}} \cong E_{2} / \mathbb{F}_{2^{m}}$, then we have $a_{6}=\bar{a}_{6}$ and the isomorphism is given by

$$
\begin{aligned}
\phi_{s}: E_{1} & \longrightarrow E_{2}, \\
(x, y) & \longmapsto(x, y+s x),
\end{aligned}
$$

where $s$ is an element in $\mathbb{F}_{2^{m}}$ and satisfies the equation

$$
s^{2}+s=a_{2}+\bar{a}_{2}
$$

(see [12, Section 3.3]).

Remark that if $s_{1}$ is a solution of (8) then $s_{2}:=s_{1}+1$ is the other solution. Furthermore, $\phi_{s}$ is an automorphism if and only if $s \in\{0,1\}$ ([13, Appendix A, Proposition 1.2]).

\section{Metrics}

In this section we assume that $K=\mathbb{F}_{2^{m}}$ for $m \geq 2$. We consider the set of nonsupersingular elliptic curves over $\mathbb{F}_{2^{m}}$ in simplified Weierstrass form; namely,

$$
\begin{aligned}
& \mathscr{E}(m) \\
& :=\left\{E / \mathbb{F}_{2^{m}}: \text { elliptic curve } \mid E \text { is of the form }(5)\right\} .
\end{aligned}
$$

Throughout this section, we assume that $E_{i} / \mathbb{F}_{2^{m}} \in \mathscr{E}(m)$ are elliptic curves for $i=1,2,3$. In addition, we denote

$$
\begin{aligned}
& \phi_{s}: E_{1} \longrightarrow E_{2}, \\
&(x, y) \longmapsto(x, y+s x), \\
& \phi_{t}: E_{2} \longrightarrow E_{3}, \\
&(x, y) \longmapsto(x, y+t x), \\
& \phi_{u}: E_{1} \longrightarrow E_{3}, \\
&(x, y) \longmapsto(x, y+u x),
\end{aligned}
$$


if $E_{1} / \mathbb{F}_{2^{m}} \cong E_{2} / \mathbb{F}_{2^{m}}, E_{2} / \mathbb{F}_{2^{m}} \cong E_{3} / \mathbb{F}_{2^{m}}$, and $E_{1} / \mathbb{F}_{2^{m}} \cong E_{3} / \mathbb{F}_{2^{m}}$, respectively. Let $\widetilde{\mathscr{B}}$ be the set of all basis of a linear space $\mathbb{F}_{2^{m}}$ over $\mathbb{F}_{2}$. We put

$$
\begin{aligned}
\mathscr{B} & :=\left\{\mathbf{v}=\left\langle\mathbf{v}_{1}, \ldots, \mathbf{v}_{m}\right\rangle \in \widetilde{\mathscr{B}} \mid \text { there exists } j\right. \\
& \left.\in\{1, \ldots, m\} \text { such that } \mathbf{v}_{j}=1\right\},
\end{aligned}
$$

where $1 \in \mathbb{F}_{2^{m}}$ is the multiplicative identity element. Note that the set $\mathscr{B}$ is a nonempty finite set because a polynomial basis belongs to the set $\mathscr{B}$. We choose a basis $\mathbf{v}=\left\langle\mathbf{v}_{1}, \ldots, \mathbf{v}_{m}\right\rangle \in \mathscr{B}$ and fixed it. Then there exists $j_{0} \in\{1, \ldots, m\}$ such that $\mathbf{v}_{j_{0}}=1$. Let

$$
\begin{array}{ccc}
f_{j_{0}}: \quad \mathbb{F}_{2^{m}} & \longrightarrow & \mathbb{F}_{2}^{m-1} \\
\Psi & \Psi \\
x= & \sum_{i=1}^{m} x_{i} \mathbf{v}_{i} \longmapsto f_{j_{0}}(x)=\widehat{x}_{j_{0}}=\left(x_{1}, \ldots, x_{j_{0}-1}, x_{j_{0}+1}, \ldots, x_{m}\right)
\end{array}
$$

denote the surjective $\mathbb{F}_{2}$-linear map, where $x_{i} \in \mathbb{F}_{2}(1 \leq i \leq$ $m)$. For any $x=\sum_{i=1}^{m} x_{i} \mathbf{v}_{i} \in \mathbb{F}_{2^{m}}\left(x_{i} \in \mathbb{F}_{2}\right)$, we write

$$
\begin{aligned}
\operatorname{wt}\left(f_{j_{0}}(x)\right) & =w t\left(\widehat{x}_{j_{0}}\right) \\
& :=\sharp\left\{i \in\{1, \ldots, m\} \mid i \neq j_{0}, x_{i} \neq 0\right\} .
\end{aligned}
$$

Note that, for all $x \in \mathbb{F}_{2^{m}}$, we always have $\operatorname{wt}\left(f_{j_{0}}(x)\right) \leq m-1$. We put $s=\sum_{i=1}^{m} s_{i} \mathbf{v}_{i} \in \mathbb{F}_{2^{m}}\left(s_{i} \in \mathbb{F}_{2}\right), t=\sum_{i=1}^{m} t_{i} \mathbf{v}_{i} \in \mathbb{F}_{2^{m}}\left(t_{i} \in\right.$ $\left.\mathbb{F}_{2}\right)$, and $u=\sum_{i=1}^{m} u_{i} \mathbf{v}_{i} \in \mathbb{F}_{2^{m}}\left(u_{i} \in \mathbb{F}_{2}\right)$ for the isomorphisms of form (10).

Now we define the function $d_{\mathbf{v}}^{(m)}: \mathscr{E}(m) \times \mathscr{E}(m) \rightarrow \mathbb{R}$ as follows:

$$
\begin{aligned}
& d_{\mathbf{v}}^{(m)}: \mathscr{E}(m) \times \mathscr{E}(m) \longrightarrow \quad \mathbb{R} \\
& \Psi \quad \Psi \\
& \left(E_{1}, E_{2}\right) \quad \longmapsto \operatorname{wt}\left(f_{j_{0}}(s)\right) \text { if } E_{1} / \mathbb{F}_{2^{m}} \cong E_{2} / \mathbb{F}_{2^{m}}, \\
& \left(E_{1}, E_{2}\right) \longmapsto m \text { otherwise. }
\end{aligned}
$$

Remark that the function $d_{\mathbf{v}}^{(m)}$ is well-defined because, for two solutions $s_{1}, s_{2}\left(s_{2}=s_{1}+1\right)$ of (8), we have $\operatorname{wt}\left(f_{j_{0}}\left(s_{1}\right)\right)=$ $\operatorname{wt}\left(f_{j_{0}}\left(s_{2}\right)\right)$. Namely, the function $d_{\mathbf{v}}^{(m)}$ does not depend on the choice of isomorphisms.

We are ready to state and prove the main result of this paper, namely, Theorem 5, which states that the set of nonsupersingular elliptic curves over $\mathbb{F}_{2^{m}}$ in simplified Weierstrass form is a metric space under the metric $d_{\mathbf{v}}^{(m)}$.

Theorem 5 (metric on $\mathscr{E}(m))$. ( $\left.\mathscr{E}(m), d_{\mathbf{v}}^{(m)}\right)$ is a metric space.

Proof. We prove the nonnegativity, the nondegeneracy, the symmetry, and the triangular inequality.

(1) Nonnegativity. By the definition of the function $d_{\mathbf{v}}^{(m)}$, we have $d_{\mathbf{v}}^{(m)}\left(E_{1}, E_{2}\right) \geq 0$ for all $E_{1}, E_{2} \in \mathscr{E}(m)$.

(2) Nondegeneracy. Suppose that $d_{\mathbf{v}}^{(m)}\left(E_{1}, E_{2}\right)=0$. Since $m \geq$ 2 , we must have $\operatorname{wt}\left(f_{j_{0}}(s)\right)=\operatorname{wt}\left(\widehat{s}_{j_{0}}\right)=0$. This implies that $s_{i}=$ 0 for all $i \neq j_{0}$. Remember that $s_{i} \in \mathbb{F}_{2}=\{0,1\}$. Setting $s_{j_{0}}=0$ (resp., $s_{j_{0}}=1$ ) yields $s=0$ (resp., $s=1$ ). When $s=0$ or $s=1$, the isomorphism of form (10) is an automorphism. Thus,
$E_{1}=E_{2}$. Conversely, if $E_{1}=E_{2}$, then the isomorphism of form (10) is an automorphism. It then follows that $s=0,1$. Hence $s_{i}=0$ for all $i \neq j_{0}$ and therefore $d_{\mathbf{v}}^{(m)}\left(E_{1}, E_{2}\right)=$ $\operatorname{wt}\left(f_{j_{0}}(s)\right)=0$.

(3) Symmetry. If $E_{1} / \mathbb{F}_{2^{m}} \not E_{2} / \mathbb{F}_{2^{m}}$, then $d_{\mathbf{v}}^{(m)}\left(E_{1}, E_{2}\right)=$ $d_{\mathbf{v}}^{(m)}\left(E_{2}, E_{1}\right)=m$. Otherwise, we define

$$
\begin{aligned}
& \psi: E_{2} \longrightarrow E_{1}, \\
& (x, y) \longmapsto(x, y+s x) .
\end{aligned}
$$

One can easily check that $\psi$ is an isomorphism. Thus $d_{\mathbf{v}}^{(m)}\left(E_{1}, E_{2}\right)=d_{\mathbf{v}}^{(m)}\left(E_{2}, E_{1}\right)=\operatorname{wt}\left(\widehat{s}_{j_{0}}\right)$.

(4) Triangular Inequality. Let $E_{1}, E_{2}, E_{3} \in \mathscr{E}(m)$. We claim that

$$
d_{\mathbf{v}}^{(m)}\left(E_{1}, E_{3}\right) \leq d_{\mathbf{v}}^{(m)}\left(E_{1}, E_{2}\right)+d_{\mathbf{v}}^{(m)}\left(E_{2}, E_{3}\right) .
$$

There are two cases to consider. Namely, $E_{1} / \mathbb{F}_{2^{m}} \not \equiv E_{3} / \mathbb{F}_{2^{m}}$ and $E_{1} / \mathbb{F}_{2^{m}} \cong E_{3} / \mathbb{F}_{2^{m}}$.

Case $1\left(E_{1} / \mathbb{F}_{2^{m}} \not E_{3} / \mathbb{F}_{2^{m}}\right)$. In this case, we have $d_{\mathbf{v}}^{(m)}\left(E_{1}, E_{3}\right)=$ $m$. If follows immediately from $E_{1} / \mathbb{F}_{2^{m}} \not E_{3} / \mathbb{F}_{2^{m}}$ that $E_{1} / \mathbb{F}_{2^{m}} \not E_{2} / \mathbb{F}_{2^{m}}$ or $E_{2} / \mathbb{F}_{2^{m}} \not E_{3} / \mathbb{F}_{2^{m}}$. Then $d_{\mathbf{v}}^{(m)}\left(E_{1}, E_{2}\right)=$ $m$ or $d_{\mathbf{v}}^{(m)}\left(E_{2}, E_{3}\right)=m$. This shows that $d_{\mathbf{v}}^{(m)}\left(E_{1}, E_{3}\right) \leq$ $d_{\mathbf{v}}^{(m)}\left(E_{1}, E_{2}\right)+d_{\mathbf{v}}^{(m)}\left(E_{2}, E_{3}\right)$ as claimed.

Case $2\left(E_{1} / \mathbb{F}_{2^{m}} \cong E_{3} / \mathbb{F}_{2^{m}}\right)$. There are two possibilities: $E_{1} / \mathbb{F}_{2^{m}} \not E_{2} / \mathbb{F}_{2^{m}}$ and $E_{1} / \mathbb{F}_{2^{m}} \cong E_{2} / \mathbb{F}_{2^{m}}$. The former case gives $d_{\mathbf{v}}^{(m)}\left(E_{1}, E_{2}\right)=m$, which shows that $d_{\mathbf{v}}^{(m)}\left(E_{1}, E_{3}\right) \leq$ $d_{\mathbf{v}}^{(m)}\left(E_{1}, E_{2}\right) \leq d_{\mathbf{v}}^{(m)}\left(E_{1}, E_{2}\right)+d_{\mathbf{v}}^{(m)}\left(E_{2}, E_{3}\right)$ (or, more precisely, $d_{\mathbf{v}}^{(m)}\left(E_{1}, E_{2}\right)<d_{\mathbf{v}}^{(m)}\left(E_{1}, E_{2}\right)+d_{\mathbf{v}}^{(m)}\left(E_{2}, E_{3}\right)$ since $E_{2} / \mathbb{F}_{2^{m}} \not \equiv$ $\left.E_{3} / \mathbb{F}_{2^{m}}\right)$. In the latter case, we have $\phi_{u}=\phi_{t} \circ \phi_{s}$. This yields that $d_{\mathbf{v}}^{(m)}\left(E_{1}, E_{2}\right)=\operatorname{wt}\left(f_{j_{0}}(s)\right), d_{\mathbf{v}}^{(m)}\left(E_{2}, E_{3}\right)=\operatorname{wt}\left(f_{j_{0}}(t)\right)$, and $d_{\mathbf{v}}^{(m)}\left(E_{1}, E_{3}\right)=\operatorname{wt}\left(f_{j_{0}}(u)\right)=w t\left(f_{j_{0}}(s+t)\right)$, respectively. Since $\left|s_{i}+t_{i}\right| \leq\left|s_{i}\right|+\left|t_{i}\right|$ for all $i \in\{1, \ldots, m\}, i \neq j_{0}$, we obtain

$$
\begin{aligned}
\left|w t\left(f_{j_{0}}(u)\right)\right| & =\left|w t\left(f_{j_{0}}(s+t)\right)\right| \\
& \leq\left|w t\left(f_{j_{0}}(s)\right)\right|+\left|w t\left(f_{j_{0}}(t)\right)\right|,
\end{aligned}
$$


where $\left|s_{i}\right|$ (resp., $\left|t_{i}\right|$ ) is equal to 1 when $s_{i}=1$ (resp., $t_{i}=$ 1 ) and otherwise $\left|s_{i}\right|$ (resp., $\left|t_{i}\right|$ ) is equal to 0 . Hence we have $d_{\mathbf{v}}^{(m)}\left(E_{1}, E_{3}\right) \leq d_{\mathbf{v}}^{(m)}\left(E_{1}, E_{2}\right)+d_{\mathbf{v}}^{(m)}\left(E_{2}, E_{3}\right)$. This completes the proof.

Remark 6. The main observation of Theorem 5 is that the isomorphism of form (10) is an automorphism if and only if $s \in\{0,1\}$. By omitting the $j_{0}$ th entry of $s$ and by summing up the number of nonzero $s_{i}$ with $1 \leq i \leq m$ and $i \neq j_{0}$, one can construct a metric on $\mathscr{E}(m)$. In order to omit the $j_{0}$ th entry, we use a basis $\mathbf{v}$ which is belonging to the set $\mathscr{B}$. The nonnegativity and the symmetry for $d_{\mathbf{v}}^{(m)}$ are obvious. The nondegeneracy for $d_{\mathbf{v}}^{(m)}$ is followed by the omission of $j_{0}$ th entry. The triangular inequality for the Hamming distance implies the triangular inequality for $d_{\mathbf{v}}^{(m)}$.

In the definition of $d_{\mathbf{v}}^{(m)}$, we put $d_{\mathbf{v}}^{(m)}\left(E_{1}, E_{2}\right)=m$ when $E_{1} / \mathbb{F}_{2^{m}} \not E_{2} / \mathbb{F}_{2^{m}}$. However, the value $m$ does not have any special meanings, and one can use any other positive integer greater than or equal to $m$ in order to define different metrics on $\mathscr{E}(m)$.

Corollary 7 (other metrics on $\mathscr{E}(m)$ ). (1) For any integer $n \in$ $\mathbb{Z}$ greater than or equal to $m$, define the function $d_{\mathbf{v}}^{(n)}: \mathscr{E}(m) \times$ $\mathscr{E}(m) \rightarrow \mathbb{R}$ as follows:

$$
\begin{aligned}
& d_{\mathbf{v}}^{(n)}: \mathscr{E}(m) \times \mathscr{E}(m) \longrightarrow \quad \mathbb{R} \\
& \Psi \quad \psi \\
& \left(E_{1}, E_{2}\right) \quad \longmapsto \operatorname{wt}\left(f_{j_{0}}(s)\right) \text { if } E_{1} / \mathbb{F}_{2^{m}} \cong E_{2} / \mathbb{F}_{2^{m}} \text {, } \\
& \left(E_{1}, E_{2}\right) \longmapsto n \text { otherwise. }
\end{aligned}
$$

Then $\left(\mathscr{E}(m), d_{\mathbf{v}}^{(n)}\right)$ is a metric space.

(2) We define the function $d_{\mathbf{v}}^{(\infty)}: \mathscr{E}(m) \times \mathscr{E}(m) \rightarrow \mathbb{R} \cup\{\infty\}$ as follows:

$$
\begin{aligned}
& d_{\mathbf{v}}^{(\infty)}: \mathscr{E}(m) \times \mathscr{E}(m) \longrightarrow \mathbb{R} \cup\{\infty\} \\
& \Psi \quad \Psi \\
& \left(E_{1}, E_{2}\right) \quad \longmapsto w t\left(f_{j_{0}}(s)\right) \text { if } E_{1} / \mathbb{F}_{2^{m}} \cong E_{2} / \mathbb{F}_{2^{m}} \text {, } \\
& \left(E_{1}, E_{2}\right) \longmapsto \infty \quad \infty \quad \text { otherwise. }
\end{aligned}
$$

Then $\left(\mathscr{E}(m), d_{\mathbf{v}}^{(\infty)}\right)$ is also a metric space.

(3) Set

$$
\mathcal{N}_{m}:=\{m, m+1, m+2, \ldots\} \cup\{\infty\} .
$$

We choose a subset $\mathcal{S} \subseteq \mathscr{B}$. Since $\mathscr{B}$ is a finite set, the subset $\mathcal{S}$ is also a finite set. For each $\mathbf{v} \in \mathcal{S}$, we take $n_{\mathbf{v}} \in \mathcal{N}_{m}$. Then the function

$$
\begin{aligned}
\sum_{\mathbf{v} \in \mathcal{S}} d_{\mathbf{v}}^{\left(n_{\mathbf{v}}\right)}: \mathscr{E}(m) \times \mathscr{E}(m) & \longrightarrow \mathbb{R} \cup\{\infty\}, \\
\left(E_{1}, E_{2}\right) & \longmapsto \sum_{\mathbf{v} \in \mathcal{S}} d_{\mathbf{v}}^{\left(n_{\mathbf{v}}\right)}\left(E_{1}, E_{2}\right)
\end{aligned}
$$

is also a metric on $\mathscr{E}(m)$.
(4) We put $\mathbb{R}_{>0}:=\{c \in \mathbb{R} \mid c>0\}$. For each $\mathbf{v} \in \mathcal{S}$, we take $c_{\mathbf{v}}^{\left(n_{\mathrm{v}}\right)} \in \mathbb{R}_{>0}$. Then as in (3), we can define the metric

$$
\begin{aligned}
\sum_{\mathbf{v} \in \mathcal{S}} c_{\mathbf{v}}^{\left(n_{\mathrm{v}}\right)} d_{\mathbf{v}}^{\left(n_{\mathbf{v}}\right)}: \mathscr{E}(m) \times \mathscr{E}(m) & \longrightarrow \mathbb{R} \cup\{\infty\} \\
\left(E_{1}, E_{2}\right) & \longmapsto \sum_{\mathbf{v} \in \mathcal{S}} c_{\mathbf{v}}^{\left(n_{\mathbf{v}}\right)} d_{\mathbf{v}}^{\left(n_{\mathbf{v}}\right)}\left(E_{1}, E_{2}\right)
\end{aligned}
$$

on $\mathscr{E}(m)$.

Proof. The proofs are very similar to the proof of Theorem 5; thus we omit them.

Remark 8 (topological properties of a metric space $\mathscr{E}(\mathrm{m})$ ). We recall that a metric space gives rise to a topology. Here, we make sure of the properties of the topology on $\mathscr{E}(m)$ induced by a metric. Given a metric space $(\mathscr{E}(m), d)$, let $\mathcal{O}_{d}$ be the topology on $\mathscr{E}(m)$ induced by the metric $d$. Note that the facts shown below do not depend on the metric $d$. A metric space is a Hausdorff space [14, p. 110, Proposition 11.5]. A finite subset of a topological space is compact [14, p. 128, Example 13.8(a)]. These two facts indicate that $\left(\mathscr{E}(m), \mathscr{O}_{d}\right)$ is a compact Hausdorff space. If a finite space is Hausdorff then its topology is discrete [14, p. 111, Exercise 11.2(b)]. Therefore $\widehat{O}_{d}$ is discrete, and hence $\left(\mathscr{E}(m), \mathcal{O}_{d}\right)$ is totally disconnected. Any finite metric space is compact, therefore $\left(\mathscr{E}(m), \sigma_{d}\right)$ is a complete metric space.

\section{Conclusion}

In this paper, we have defined some metrics on the sets of nonsupersingular elliptic curves in simplified Weierstrass form over finite fields of characteristic two. In order to derive analogous results for the case of supersingular elliptic curves of characteristic two and for the case of elliptic curves of characteristic three, some deep observation on the properties of elliptic curves over finite fields will be needed.

\section{Conflict of Interests}

The author declares that there is no conflict of interests regarding the publication of this paper.

\section{Acknowledgment}

This work was supported by a grant for young researchers from Shimane University in 2015.

\section{References}

[1] N. Koblitz, "Elliptic curve cryptosystems," Mathematics of Computation, vol. 48, no. 177, pp. 203-209, 1987.

[2] V. S. Miller, "Use of elliptic curves in cryptography," in Advances in Cryptology-CRYPTO '85 Proceedings, vol. 218 of Lecture Notes in Computer Science, pp. 417-426, Springer, Berlin, Germany, 1986.

[3] D. Boneh and M. Franklin, "Identity based encryption from the Weil pairing," SIAM Journal on Computing, vol. 32, no. 3, pp. 586-615, 2003. 
[4] D. Boneh, B. Lynn, and H. Shacham, "Short signatures from the Weil pairing," Journal of Cryptology, vol. 17, no. 4, pp. 297-319, 2004.

[5] A. O. L. Atkin and F. Morain, "Elliptic curves and primality proving," Mathematics of Computation, vol. 61, no. 203, pp. 2968, 1993.

[6] S. Goldwasser and J. Kilian, "Primality testing using elliptic curves," Journal of the ACM, vol. 46, no. 4, pp. 450-472, 1999.

[7] H. W. Lenstra Jr., "Factoring integers with elliptic curves," Annals of Mathematics, vol. 126, no. 3, pp. 649-673, 1987.

[8] P. L. Montgomery, "Speeding the Pollard and elliptic curve methods of factorization," Mathematics of Computation, vol. 48, no. 177 , pp. 243-264, 1987.

[9] P. K. Mishra and K. C. Gupta, "A metric on the set of elliptic curves over $\mathbb{F}_{p}$," Applied Mathematics Letters, vol. 21, no. 12, pp. 1330-1332, 2008.

[10] F. Vetro, "Metrics on the set of elliptic curves over $\mathbb{F}_{p}$," International Journal of Contemporary Mathematical Sciences, vol. 1, no. 1, pp. 22-24, 2011.

[11] M. Joye and C. Tymen, "Protections against differential analysis for elliptic curve cryptography: an algebraic approach," in Cryptographic Hardware and Embedded Systems-CHES 2001 Proceedings, vol. 2162 of Lecture Notes in Computer Science, pp. 377-390, Springer, Berlin, Germany, 2011.

[12] A. Menezes, Elliptic Curve Public Key Cryptosystems, Kluwer Academic Publishers, Boston, Mass, USA, 1993.

[13] J. H. Silverman, The Arithmetic of Elliptic Curves, vol. 106 of Graduate Texts in Mathematics, Springer, New York, NY, USA, 2nd edition, 2009.

[14] W. A. Sutherland, Introduction to Metric and Topological Spaces, Oxford University Press, 2nd edition, 2009. 


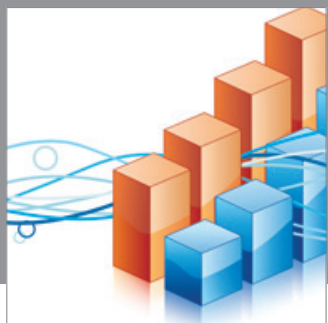

Advances in

Operations Research

mansans

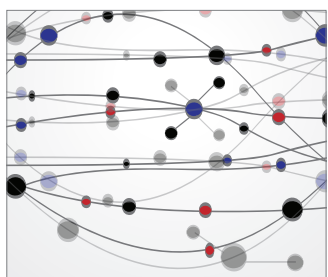

The Scientific World Journal
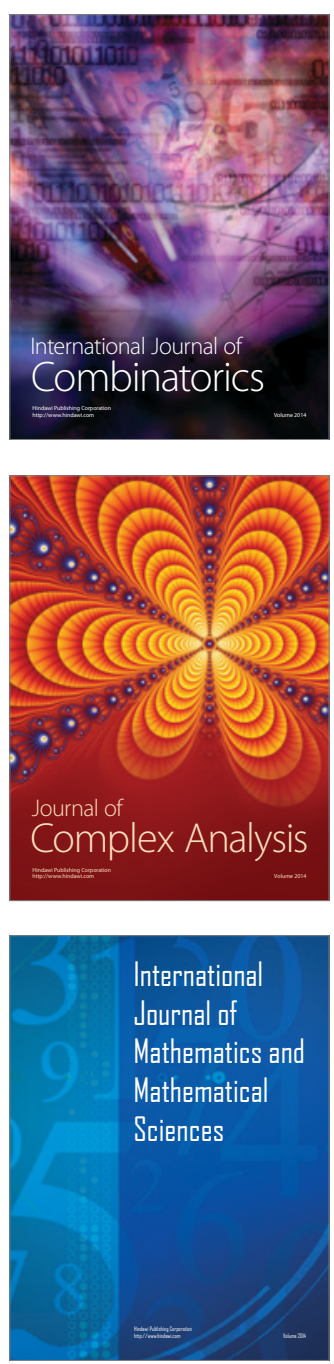
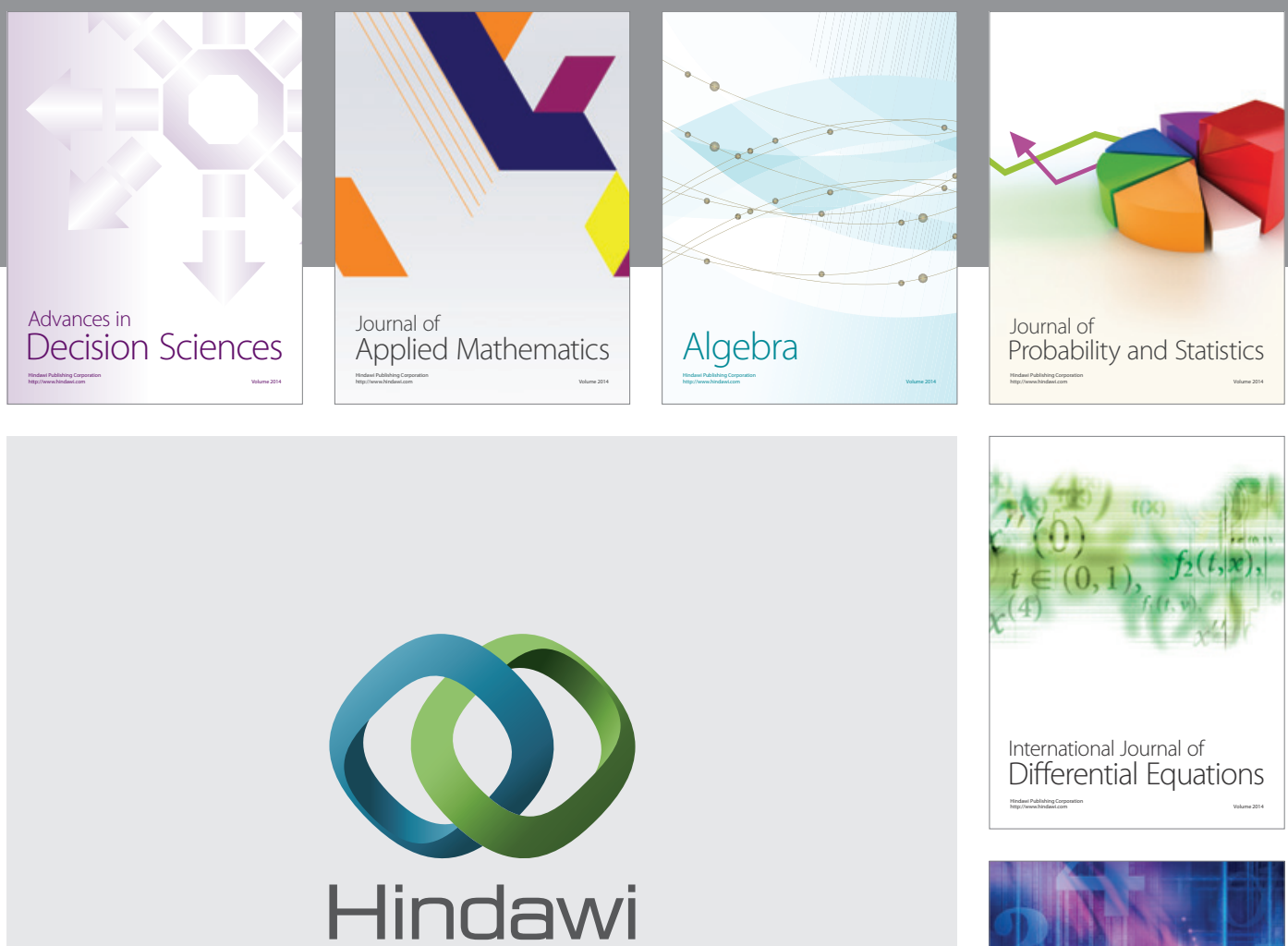

Submit your manuscripts at http://www.hindawi.com
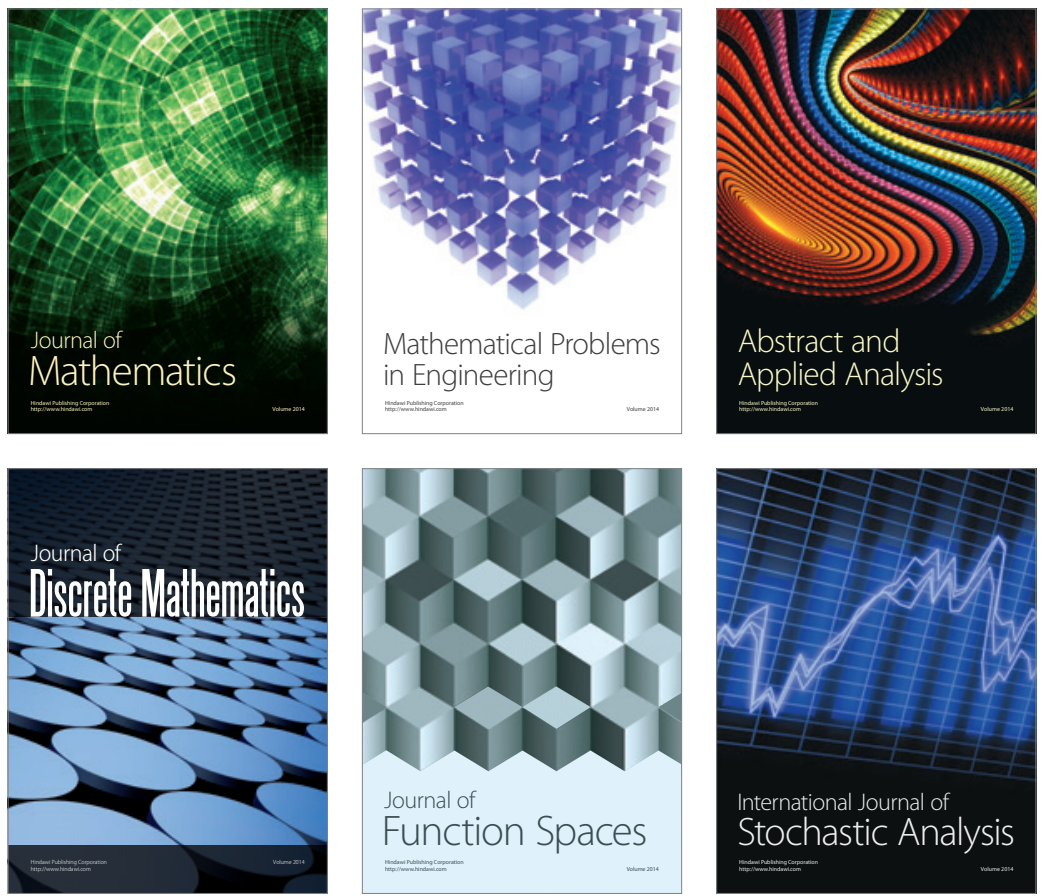

Journal of

Function Spaces

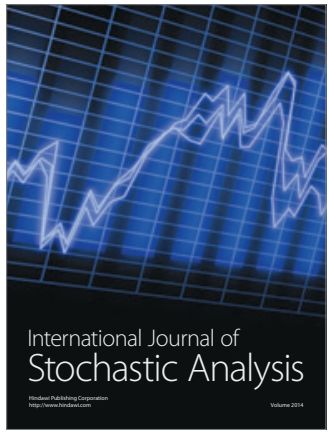

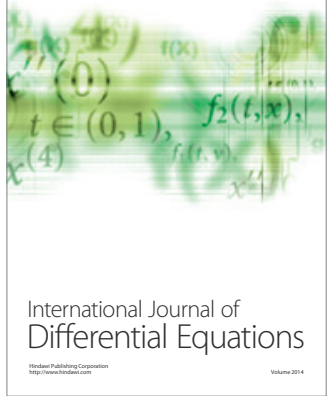
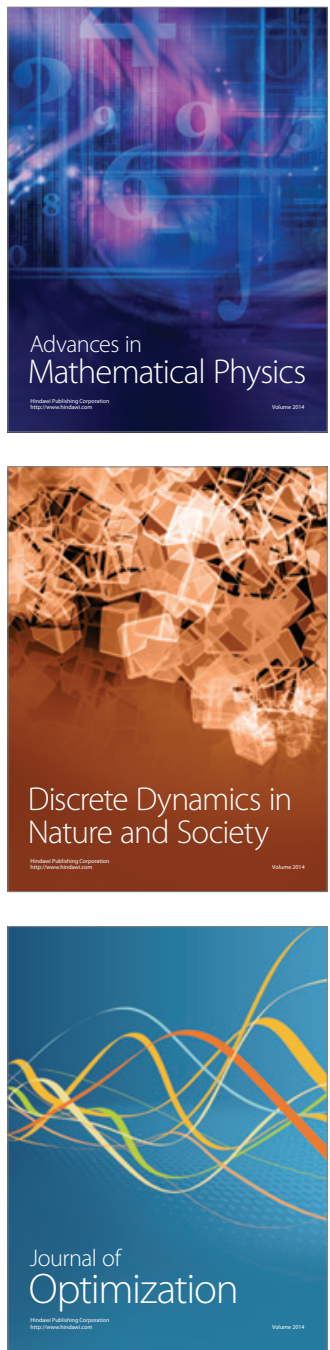